Reprod. Nutr. Dévelop., 1988, 28 (1), 115-116.

\title{
Influence d'un apport d'orge floconnée ou de pulpe de betterave à une herbe exploitée au stade épiaison sur la digestion dans le rumen et le flux d'azote non ammoniacal au duodénum chez le mouton
}

\author{
F. RODRIGUEZ, A. THÉWIS, Nicole BARTIAUX-THILL $\left(^{*}\right)$, J. J. FERNANDEZ, \\ E. FRANÇOIS (*)
}

Faculté des Sciences Agronomiques de l'Etat, Service de Zootechnie, Passage des Déportés, 2, B 5800 Gembloux, Belgique.

(*) Centre de Recherches Agronomiques de l'Etat,

Avenue de la Faculté d'Agronomie, 22, B 5800 Gembloux, Belgique.

Summary. The influence of barley and sugar beet pulp on the digestibility and duodenal $\mathrm{N}$ flow of a grass-base diet was studied in stall-fed sheep. Both supplements affected digestibility of the diet and increased duodenal non ammonia nitrogen flow.

Nous avons observé récemment (Bartiaux-Thill et al., 1987 ; Théwis et al., 1987) une influence spécifique de l'orge floconnée et de la pulpe de betterave sur la digestion d'une ration à base d'herbe fraîche exploitée au stade montaison chez le bélier et notamment une augmentation du flux d'azote non ammoniacal (NNA) au duodénum en cas de supplémentation par la pulpe. Nous présentons ici les résultats partiels d'une expérience semblable réalisée avec une herbe exploitée au stade épiaison.

Matériel et méthodes. Douze béliers adultes, porteurs d'une canule du rumen et d'une canule simple au duodénum, répartis en trois lots homogènes, reçoivent l'un des trois régimes suivants: herbe (H), herbe plus orge floconnée (HO) ou herbe plus pulpe de betterave déshydratée (HP). L'herbe fauchée chaque jour, provient d'une prairie permanente à dominance de ray-grass anglais exploitée au stade pleine épiaison $(11,8 \%$ de matières azotées (MA) et $24,7 \%$ de cellulose brute). Les suppléments représentent $35 \%$ de la matière sèche (MS). La ration est distribuée à raison de $60 \mathrm{~g} \mathrm{MS} / \mathrm{kg} \mathrm{P0,75/jour} \mathrm{en} \mathrm{quatre} \mathrm{repas.} \mathrm{Après} \mathrm{une}$ période d'adaptation de deux semaines, les animaux font l'objet d'une mesure de

Travaux subsidiés par le Fonds de la Recherche Fondamentale Collective, convention 2.4542.84, par la Communauté Economique Européenne, contrat n TSDA-189 B (TT) et par le Ministère de l'Emploi et du Travail contrat TCT $\mathrm{n}^{\circ} 8659$. 
digestibilité (7 jours). Pendant les trois jours suivants, on prélève, au moment des repas, un échantillon de chyme duodénal dont le flux journalier est déterminé au moyen de $\mathrm{Cr}_{2} \mathrm{O}_{3}$. Enfin, pendant deux jours, des échantillons de jus de rumen sont prélevés $1 \mathrm{~h} 1 / 2$ après les repas de $9 \mathrm{~h}$ et $21 \mathrm{~h}$ en vue de mesurer le pH et la concentration en azote ammoniacal $\left(\mathrm{N}-\mathrm{NH}_{3}\right)$.

Résultats et discussion. L'orge et dans une mesure moindre la pulpe améliorent la digestibilité de la matière organique (dMO) de la ration indépendamment de tout effet d'associativité (différences entre les digestibilités mesurées et attendues, ces dernières étant calculées en prenant les digestibilités de l'orge et de la pulpe données par Demarquilly, Andrieu et Sauvant, 1978 ; tabl. 1). Cela est dû à la dMO plus élevée des suppléments que celle de l'herbe.

TABL. 1. - Digestion de la matière organique (MO) et des matières azotées pour les régimes herbe (H), herbe + orge (HO) et herbe + pulpe (HP).

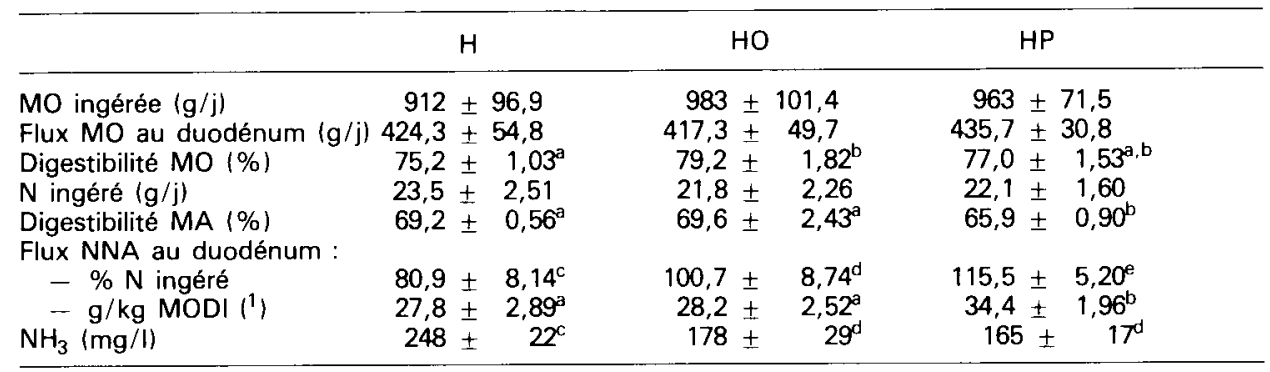

(1) MODI = matière organique digestible ingérée ; $\mathrm{a}, \mathrm{b}: \alpha \leqslant 0,05 ; \mathrm{c}, \mathrm{d}, \mathrm{e}: \alpha \leqslant 0,01$.

En ce qui concerne la digestibilité des matières azotées (dMA), contrairement à l'orge, la pulpe, caractérisée par une dMA très faible - $52 \%$ - (Demarquilly, Andrieu et Sauvant, 1978), engendre une diminution significative de la dMA de la ration bien que les quantités d'azote ingérées soient sensiblement égales pour les trois régimes (tabl. 1). Toutefois, l'effet d'associativité est significativement $(\alpha<0,05)$ positif $(+2,59)$.

Comme précédemment (Théwis et al., 1987), les suppléments et spécialement la pulpe diminuent significativement la concentration en ammoniac dans le rumen, par ailleurs très élevée pour une herbe pauvre en MA.

Parallèlement, ils augmentent le flux journalier de NNA au duodénum (tabl. 1). Cela s'explique sans doute par une amélioration de la protéosynthèse microbienne dans le rumen en cas de supplémentation et tout particulièrement dans le cas de la pulpe.

Ces résultats confirment donc l'importance de la nature du complément énergétique comme moyen d'augmenter le flux de NNA au duodénum chez les ruminants recevant de l'herbe fraîche.

Bartiaux-Thill N., Théwis A., Rodriguez F., François E., 1987. Reprod. Nutr. Dévelop., 27, $279-280$. Demarquilly C., Andrieu J., Sauvant D., 1978. In Alimentation des ruminants. Ed. INRA Publ., Versailles, p. 519-555.

Théwis A., Lemal D., Rodriguez F., François E., Bartiaux-Thill N., Baudart E., Dardenne G., 1987. Reprod. Nutr. Dévelop., 27, 269-270. 